\title{
Microbial Products Trigger Autoimmune Ocular Inflammation
}

\author{
Chiaki Fujimoto Guangpu Shi Igal Gery \\ Laboratory of Immunology, National Eye Institute, NIH, Bethesda, Md., USA
}

\section{Key Words}

Ocular inflammation - Autoimmunity • Microbial products • Toll-like receptors $\cdot$ Toll-like receptor ligands $\cdot$ Cytokines $•$ Pertussis toxin

\begin{abstract}
Purpose: Microbial products stimulate the immune system by interacting with Toll-like receptors (TLR) on antigen-presenting cells. This study examined the hypothesis that microbial products, which function as TLR ligands, are playing a major role in triggering pathogenic autoimmunity. Methods: An experimental system was developed in which microbial TLR ligands were tested in vivo for their capacity to stimulate naïve CD4 cells specific against hen egg lysozyme (HEL) to become effector cells capable of inducing inflammation in eyes in which HEL is expressed. The ligands' mode of action was analyzed by determining their effects on the proliferation, acquisition of tissue-invading capacity, i.e. elevated CD49d and decreased CD62L expression, and production of interferon- $\gamma$ by the HEL-specific cells. Results: All the 7 tested TLR ligands triggered ocular inflammation in the experimental system used here, with pertussis toxin surpassing all other ligands in its activities. A correlation was found between the capacity of the ligands to trigger pathogenic immunity and to stimulate the proliferation, modification of cell surface and interferon- $\gamma$ production by $T$ cells. Conclusions: This study provides direct evidence to support the notion that microbial products are capable of triggering pathogenic autoimmunity.

Copyright $\odot 2008$ S. Karger AG, Basel
\end{abstract}

\section{Introduction}

The notion that microbial infection may trigger autoimmunity, suggested many years ago, was based on the observations that 'environmental factors' play a role in the susceptibility to autoimmune diseases and that in many cases, infection preceded the onset of autoimmune disease [1]. Two possible mechanisms have been proposed for the involvement of microbial infection in the initiation of pathogenic autoimmunity: (i) the 'mimicry' phenomenon and (ii) microbial products activate naïve lymphocytes specific against self-antigens to become pathogenic effector cells.

In the mimicry phenomenon, antibodies or lymphocytes specific against microbial products recognize autologous tissue molecules and react against them $[1,2]$. The phenomenon was investigated in the ocular system and several microbial molecules were identified to sufficiently cross-react with uveitogenic antigens so that immunization with these molecules initiated development of ocular inflammation identical to that induced by immunization with the ocular antigens, i.e. experimental autoimmune uveoretinitis [3-5].

The capacity of microbial products to activate naïve lymphocytes specific against self-antigens was indicated by the essential need for bacteria or their products to be added to adjuvants used for the initiation of pathogenic autoimmunity in experimental animals. The mechanism whereby microbial products activate lymphocytes has been recently clarified, following the discovery of Toll-

\section{KARGER \\ Fax +41 613061234 E-Mail karger@karger.ch} www.karger.com
Igal Gery, $\mathrm{PhD}$

National Eye Institute, NIH

Building 10, Room 10N112

Bethesda, MD 20892-1857 (USA)

Tel. +1 301496 4159, Fax +1 301480 1122, E-Mail geryi@nei.nih.gov 
like receptors (TLR) and their crucial role in triggering the innate immunity [6-8]. These data showed that the microbial molecules serve as ligands for TLR that are expressed on antigen-presenting cells (APC). The interaction between TLR and their ligands activates the APC toward maturity and acquisition of the capacity to efficiently present specific antigens to naïve $\mathrm{T}$ cells [6-8]. When the activated $\mathrm{T}$ cells are specific against self-antigens, the outcome could be an autoimmune response, with subsequent potential pathogenicity.

Studies of recent years have shown that lymphocytes specific against self-antigens escape negative selection in the thymus and enter the immune system in normal humans and animals [9-12]. In healthy conditions these self-specific cells remain naïve and harmless, but in certain circumstances, microbial infection was suggested to activate these naïve cells, via the TLR-TLR ligand system, and convert them into pathogenic effector cells. Whereas naïve cells are unable to invade nonlymphoid tissues, activated $\mathrm{T}$ cells acquire new profiles of surface markers that facilitate their invasion into such tissues $[13,14]$, where the target self-antigen is located.

In this study, we developed a new experimental system that provided direct evidence to support the hypothesis that microbial products, known to function as TLR ligands, are capable of triggering spontaneous autoimmune ocular inflammation by activating naïve $\mathrm{T}$ cells specific to self-antigens. We also used the system to gather information concerning the mode of action of the TLR ligands and the cell populations that participate in the process.

\section{Materials and Methods}

\section{Transgenic Mice}

'HEL-Tg' mice, expressing membrane-bound hen egg lysozyme (HEL) in the lens under control of the $\alpha$ A-crystallin promoter, were generated as described elsewhere [15], whereas ' $3 \mathrm{~A} 9$ ' mice, expressing HEL-specific T cell receptor (TCR) on their T lymphocytes [16], were a gift from M. Davis (Stanford University, Stanford, Calif., USA). For more detail, see Kim et al. [17] and Fujimoto et al. [18]. All procedures involving animals were performed according to the guidelines of the National Institute of Health Resolution for the Care and Use of Laboratory Animals.

\section{Reagents}

The following TLR ligands were used: a mixture of peptidoglycan (PGN) and lipoteichoic acid (LTA), zymosan, poly(I:C), lipopolysaccharide (LPS), pertussis toxin (PTX), flagellin and CpG oligodeoxynucleotide (a mixture of ODNGCTAGACGTTAGCGT and TCAACGT). For more detail, see Fujimoto et al. [18].
Adoptive Transfer of CD4 Cells and Induction of Disease

Naïve CD4 cells were isolated from spleens and lymph nodes of 3A9 mice as described in detail elsewhere [17]. Isolated CD4 cells $\left(5 \times 10^{6}\right)$ were injected via the tail vein into naïve HEL-Tg mice. On the following day, the recipient mice were injected i.v. with phosphate-buffered saline (PBS) or the TLR ligands, with or without HEL. The recipients were euthanized 7 days after cell injection. The eyes were prepared for histological analysis and the severity of inflammatory changes was evaluated as described elsewhere [17].

\section{Flow Cytometry Analysis}

Monoclonal antibodies against cell surface markers were purchased from BD Pharmingen (San Diego, Calif., USA), while a clonotypic antibody specific for the Tg TCR of the 3A9 mice, designated '1G12', was a generous gift from Emil Unanue (Washington University, St. Louis, Mo., USA). Spleen cells of recipient mice were collected and stained for surface markers using conventional methods. Flow cytometric analysis was performed on a FACS Calibur (Becton Dickinson, Franklin Lakes, N.J., USA) using FlowJo (Tree Star, Ashland, Oreg., USA).

Measurement of Cytokine Production

Spleen cells of recipient mice were cultured for $48 \mathrm{~h}$, with HEL, and cytokine levels in their supernatants were determined by the Pierce Chemical Co. (Woburn, Mass., USA), using Multiplex SearchLight ${ }^{\mathrm{TM}}$ technology.

\section{Results}

\section{The Experimental System}

To examine the capacity of TLR ligands to trigger pathogenicity in naïve $\mathrm{T}$ cells, we used an experimental system in which CD4 cells from 3A9 Tg mice, expressing TCR specific against HEL, are transferred into HEL-Tg recipient mice in which HEL is expressed in the lens (fig. 1). Severe inflammation is induced in recipient eyes following adoptive transfer of $\mathrm{T}$ cells from the $3 \mathrm{~A} 9$ donors after activation in vitro (fig. 1a; Kim et al. [17]). On the other hand, no inflammation is induced in eyes of recipient mice following adoptive transfer of as many as $5 \times 10^{6}$ naïve cells (fig. 1 b). This system thus made it possible to assess the capacity of TLR ligands to trigger pathogenicity in vivo, by treating recipients of naïve $\mathrm{T}$ cells with molecules to be tested (fig. 1c).

\section{TLR Ligands Trigger Pathogenicity by Naïve CD4 Cells}

Using the experimental system described above, we tested 7 TLR ligands for their capacity to activate naïve cells from 3A9 donors to become pathogenic and induce ocular inflammation in HEL-Tg recipients. The ligands included the combination of PGN and LTA, ligands for 


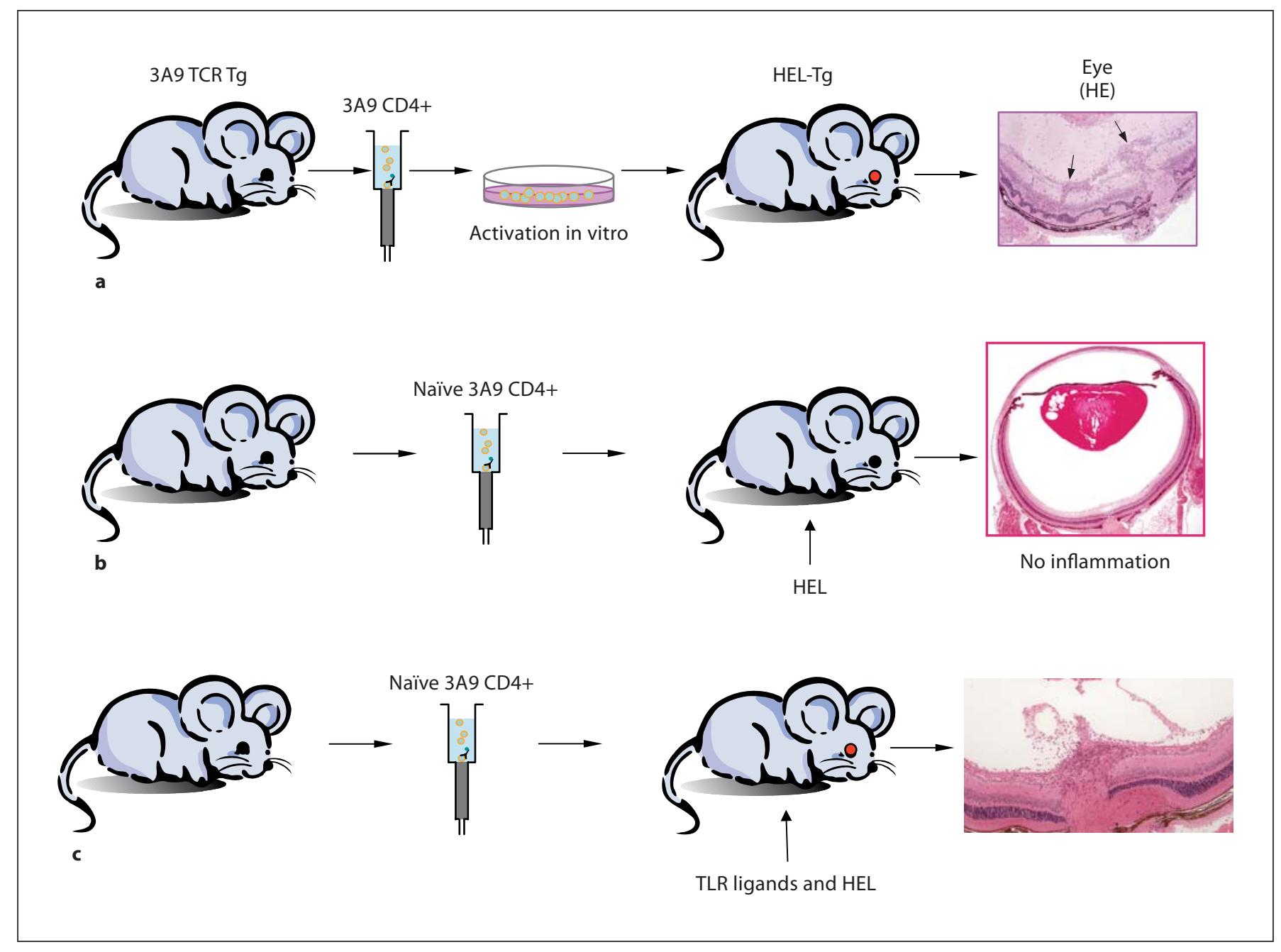

Fig. 1. The experimental system. CD4 cells from 3A9 mouse donors, with specificity toward HEL, are adoptively transferred into HEL-Tg recipients that express HEL in their lens. Ocular inflammation develops when the transferred cells are activated in vitro (a), but no changes are found when the cells are transferred at the naïve stage (b). c Experimental system used in the present study: CD4 cells are transferred at the naïve stage and are activated in vivo in mice treated with the tested TLR ligands. HEL is also injected to recipient mice in systems $\mathbf{b}$ and $\mathbf{c}$.

TLR2, zymosan, a ligand for TLR2 and 6, poly(I:C), a ligand for TLR3, LPS and PTX, ligands for TLR4, flagellin, a ligand for TLR5 and CpG, a ligand for TLR9. Data collected from repeated experiments are summarized in figure $2 \mathrm{a}$ and show that all tested ligands triggered ocular inflammation, but with differences in the severity of disease.

Typical changes in eyes of mice treated with PTX and with PGN/LTA are depicted in figure $2 \mathrm{~b}$. Moderate changes are seen in the eye of the recipient treated with PGN/LTA (PGN) and include mainly infiltration of inflammatory cells, localized at the optic nerve head and

Microbial Products Trigger Pathogenic Autoimmunity the vitreous. Also seen in this eye is infiltration of cells into the lens, the tissue where the target antigen, HEL, is produced. Dramatically severer are the changes in the eye of a PTX-treated mouse, which included severe retinal detachment, with serous exudate in the subretinal space, various degrees of damage to the retina, serous and cellular exudates in the vitreous and edema of the cornea.

Mechanisms whereby TLR Ligands Trigger Pathogenic Autoimmunity

In order to learn about the mode of action of TLR ligands in their stimulation of ocular inflammation in re- 


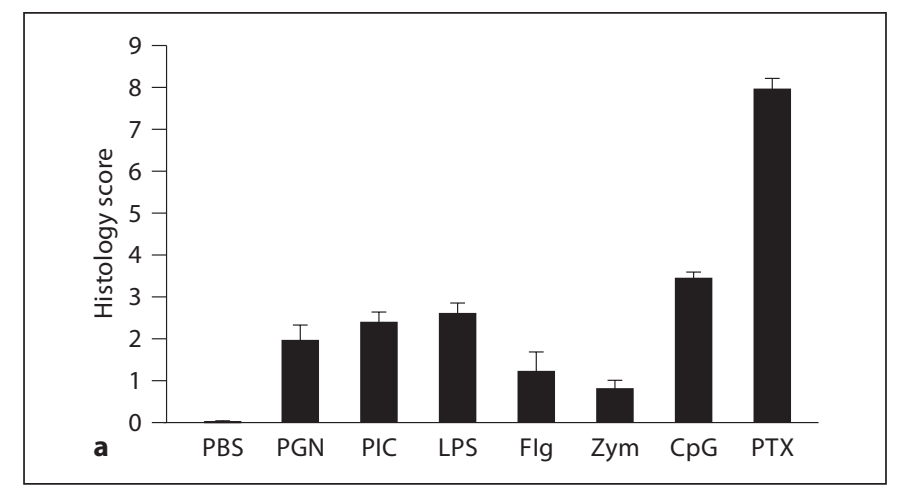

Fig. 2. Treatment with TLR ligands triggers ocular inflammation. a Recipient HEL-Tg mice were injected with $5 \times 10^{6}$ naïve $3 \mathrm{~A} 9$ CD4 cells and 1 day later were treated with TLR ligands, as indicated, along with HEL. Control mice were injected with PBS and HEL. Recipient eyes were collected 7 days after cell injection and analyzed for severity of disease, on a scale of $0-9$ [17]. Columns represent mean disease severity of 5-10 mice per group \pm SEM. b Typical histological changes in eyes of recipient mice. No changes are seen in the control mouse, treated with PBS, moderate changes are observed in the eye of a mouse treated with PGN and LTA (PGN), whereas very severe changes occur in the PTX recipient (see text for detailed description of histological changes).

cipients of naïve CD4 cells, we focused on the adoptively transferred cells and analyzed them for changes that could have contributed to the pathogenic process. We examined the transferred cells for 3 parameters, namely, their increase in number, changes in their profile of surface markers and release of a major proinflammatory cytokine, interferon (IFN)- $\gamma$.

We identified donor CD 4 cells in the recipient mice by a clonotypic antibody, 1G12, specific against the donor cells' TCR [16]. Figure 3a summarizes 5 different experiments. Treatment with all 7 ligands stimulated an increase in the number of transferred cells, but treatment with PTX stimulated an increase in donor cells which surpassed that of all other tested ligands.

The surface markers we tested were CD49d (VLA4) and CD62L (L-selectin). Studies by our group and by others showed that increase in CD49d and decrease in CD62L expression characterize tissue-invading lymphocytes $[13,14,18,19]$. As shown in figure $3 b$, donor cells from mice treated with any of the 7 TLR ligands expressed more CD49d but less CD62L than did mice of the control group.

Secretion of IFN- $\gamma$ by donor cells was determined by measuring the level of this cytokine in supernatants of cultured spleen cells of recipient mice of the different

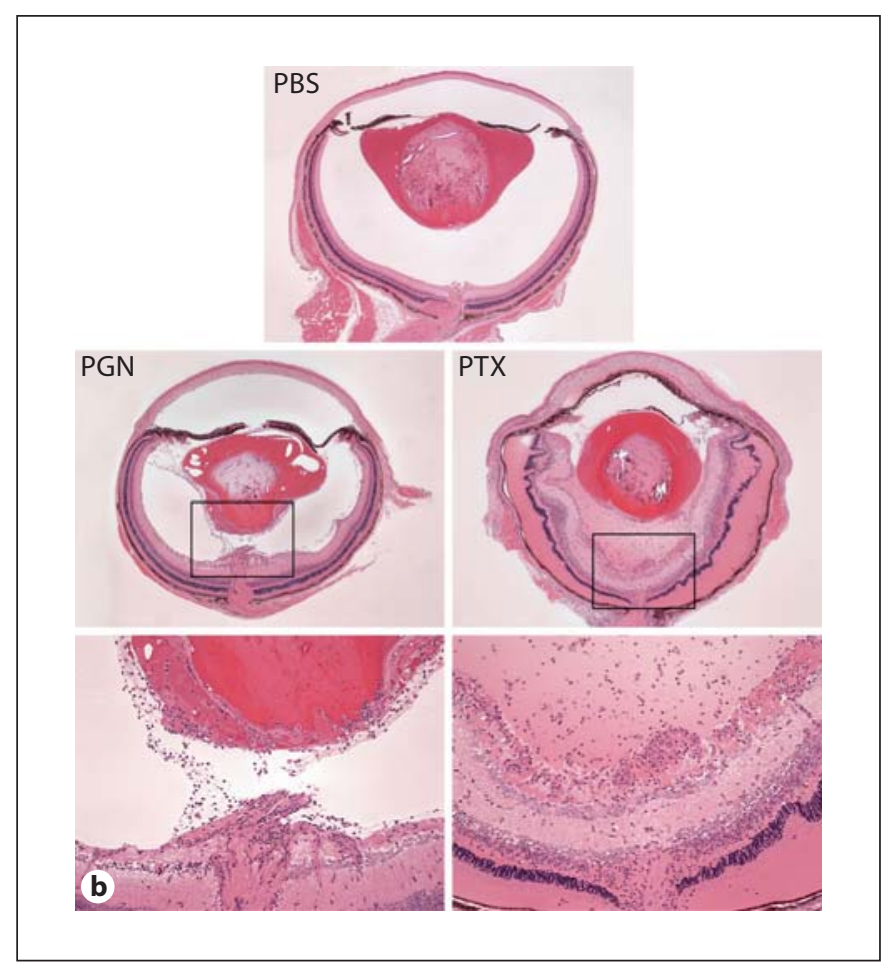

groups, stimulated with HEL; only the donor cells in these cultures recognized HEL and responded against it. The data of 3 separate experiments are summarized in figure $3 \mathrm{c}$ and demonstrate that cultures of all groups of treated mice produced more IFN- $\gamma$ than did the controls. Again, cultures of mice treated with PTX surpassed those of other groups in their release of the tested cytokine.

\section{Discussion}

The data recorded in the present study show that all tested TLR ligands are capable of triggering pathogenic autoimmunity. These experimental data thus provide direct evidence to support the notion that microbial products, while functioning as TLR ligands, may activate pathogenic autoimmunity in the eye. Furthermore, our findings for the ocular system could be applied to all other tissues in which pathogenic autoimmunity is thought to be triggered by infection.

It should be emphasized, however, that pathogenic autoimmunity is not common and is prevented in healthy individuals by several mechanisms, including T-regulatory cells $[20,21]$ and induction of anergy and peripheral tolerance $[22,23]$. In addition, the number of lympho- 

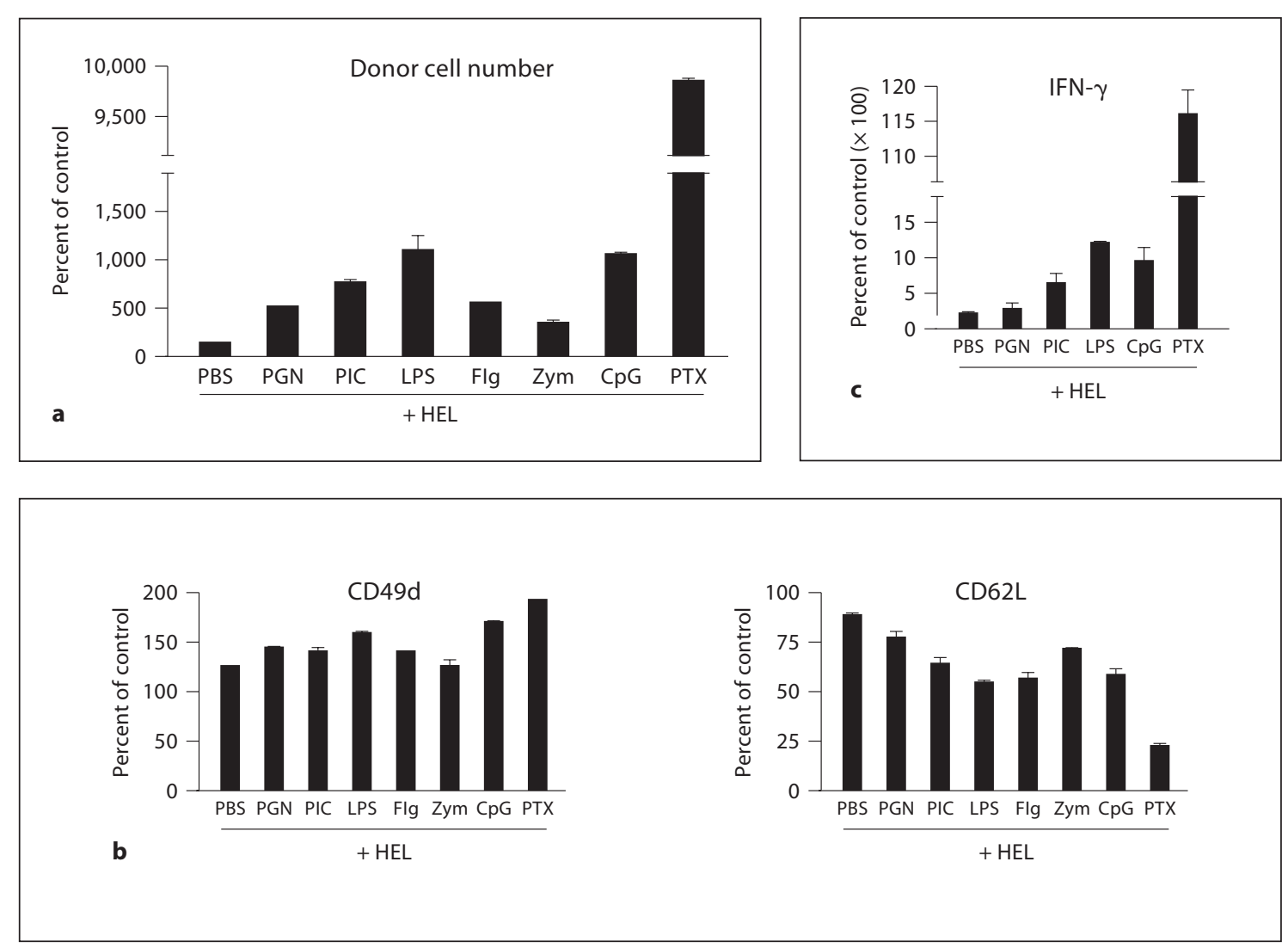

Fig. 3. Mechanisms whereby TLR ligands trigger pathogenic autoimmunity: effects on donor cells. a Increase in number of donor cells in spleen of recipient mice on day 7 after cell transfer and following treatment with TLR ligands, as indicated. Donor cells were identified by their staining with 1 G12 antibody and counted by flow cytometry. The columns represent the mean \pm SEM of 3 experiments, expressed as percent of control mice treated with PBS only. The donor cell number in control cultures was $6.8 \times$ $10^{4}$. b Changes in expression by donor cells of surface markers, CD49d and CD62L, determined by flow cytometry. The data are means \pm SEM of 3-9 separate experiments and are expressed as percent of control mice, treated with PBS only. The mean values of these controls were: CD49d, 46.9\% and CD62L, 76.9\%. c Production of IFN- $\gamma$ by cultures of recipient spleen cells following 2 days of incubation with HEL, a selective stimulant for the donor cells. The data are means \pm SEM of 3 different experiments and are expressed as percent of control recipients treated with PBS only. The level of IFN- $\gamma$ in the control cultures was $708 \mathrm{pg} / \mathrm{ml}$. $\mathrm{PIC}=$ Poly $(\mathrm{I}: \mathrm{C}) ; \mathrm{Flg}=$ flagellin; Zym = zymosan . cytes specific to self-antigens is normally smaller by far than the number used in the experimental system employed in the present study. Yet, the data collected here with the experimental system provide knowledge about the mechanisms that are likely to be involved in cases in which infection does trigger autoimmunity.

Our study focused on the involvement of T lymphocytes in the process that brings about autoimmunity following exposure to TLR ligands, but the cells directly affected by these microbial products are the APC, mainly dendritic cells and macrophages. As recorded extensively in the literature [6-8], APC stimulated by TLR ligands activate lymphocytes and convert them into effector cells capable of initiating a variety of immune responses. Our study analyzed 3 mechanisms by which treatment with TLR ligands affects T lymphocytes, i.e. intense proliferation, acquisition of new profiles of surface molecules and enhanced expression of proinflammatory cytokines.

Cell proliferation following exposure to the target antigen is a basic early step in any immune response and enhancement of the process by TLR ligands thus promotes the transferred cells' activity. This effect of TLR ligands is particularly essential for the presumptive role of the ligands in initiating autoimmunity, since the number of lymphocytes with specificity against self-antigens is normally minuscule and their expansion is crucial for their ability to trigger the pathogenic process. 
Naïve lymphocytes cannot invade nonlymphoid tissues and, therefore, initiation of the inflammatory process requires that the self-specific lymphocytes acquire a surface marker profile that facilitates tissue invasion. The most prominent changes are the increase in $\mathrm{CD} 49 \mathrm{~d}$ (VLA-4) and decrease in CD62L (L-selectin) $[13,14,18$, 19], 2 changes that took place on donor lymphocytes in mice treated with any of the tested TLR ligands.

The third mechanism whereby treatment with TLR ligands triggers pathogenic autoimmunity was enhanced production of proinflammatory cytokines. The cytokine we tested was IFN- $\gamma$ and, again, treatment with any of the tested TLR ligands was found to enhance this parameter.

Of particular interest is our finding that PTX surpassed all other tested TLR ligands in its capacity to trigger severe ocular inflammation and to initiate vigorous immune responses by the transferred $\mathrm{T}$ lymphocytes. The mode of action of PTX in enhancing immune responses is not fully understood, but our additional experiments suggest that the unique activity of PTX in the present experimental system is related in part to the capacity of this agent to selectively stimulate naïve donor CD4 lymphocytes to polarize toward the Th17 lineage. This recently discovered population of $\mathrm{T}$ cells, identified by their selective production of IL-17, has been found to play major roles in pathogenic autoimmunity [24]. Anal- ysis of eyes from PTX-treated recipients revealed that the ratio between donor cells expressing IL-17 (Th17 cells) or IFN- $\gamma$ (Th1 cells) was 5:1, whereas the ratio was $2: 1$ in recipients treated with LPS, 1:1 in poly(I:C)-treated mice and 1:2 in CpG-treated mice [Shi et al., in preparation]. Moreover, analysis of recipient spleens showed that the expression of cytokines promoting polarization toward Th17, namely IL-1 and IL-6, was exceedingly higher in recipients treated with PTX than in those treated with other tested TLR ligands [Shi et al., in preparation].

In summary, data collected by an experimental system we developed provide direct evidence showing that microbial products, functioning as TLR ligands, are capable of triggering pathogenic autoimmunity by stimulating naïve lymphocytes specific against a self-antigen to become effector cells and initiate inflammation in tissues where the target antigen is expressed. Of interest is the unusual capacity of PTX to stimulate the immune system and trigger severe inflammatory changes.

\section{Acknowledgments}

The authors thank Dr. E.F. Wawrousek for providing the HELTg mice, Dr. D.M. Klinman for providing the CpG oligodeoxynucleotide and B.P. Vistica for excellent technical assistance.

\section{References}

$\checkmark 1$ Oldstone MB: Molecular mimicry and immune-mediated diseases. FASEB J 1998;12: $1255-1265$.

-2 Barnaba V, Sinigaglia F: Molecular mimicry and $\mathrm{T}$ cell-mediated autoimmune disease. $\mathrm{J}$ Exp Med 1997;185:1529-1531.

- 3 Singh VK, Yamaki K, Abe T, Shinohara T: Molecular mimicry between uveitopathogenic site of retinal S-antigen and Escherichia coli protein: induction of experimental autoimmune uveitis and lymphocyte crossreaction. Cell Immunol 1989;122:262-273.

-4 Singh VK, Yamaki K, Donoso LA, Shinohara T: Molecular mimicry. Yeast histone H3-induced experimental autoimmune uveitis. J Immunol 1989;142:1512-1517.

5 Singh VK, Kalra HK, Yamaki K, Abe T, Donoso LA, Shinohara T: Molecular mimicry between a uveitopathogenic site of S-antigen and viral peptides. Induction of experimental autoimmune uveitis in Lewis rats. J Immunol 1990;144:1282-1287.
-6 Janeway CA Jr, Medzhitov R: Innate immune recognition. Annu Rev Immunol 2002;20: 197-216.

-7 Akira S, Takeda K: Toll-like receptor signalling. Nat Rev Immunol 2004;4:499-511.

8 Pulendran B: Variegation of the immune response with dendritic cells and pathogen recognition receptors. J Immunol 2005; 174: 2457-2465.

\$ Walker LS, Abbas AK: The enemy within: keeping self-reactive $\mathrm{T}$ cells at bay in the periphery. Nat Rev Immunol 2002;2:11-19.

-10 Starr TK, Jameson SC, Hogquist KA: Positive and negative selection of T cells. Annu Rev Immunol 2003;21:139-176.

11 Waldner H, Collins M, Kuchroo VK: Activation of antigen-presenting cells by microbial products breaks self tolerance and induces autoimmune disease. J Clin Invest 2004;113: 990-997.

12 Danke NA, Koelle DM, Yee C, Beheray S, Kwok WW: Autoreactive T cells in healthy individuals. J Immunol 2004; 172:59675972.
13 Sallusto F, Lenig D, Forster R, Lipp M, Lanzavecchia A: Two subsets of memory T lymphocytes with distinct homing potentials and effector functions. Nature 1999;401: 708-712.

14 Siegelman MH, Stanescu D, Estess P: The CD44-initiated pathway of T-cell extravasation uses VLA- 4 but not LFA- 1 for firm adhesion. J Clin Invest 2000;105:683-691.

15 Lai JC, Fukushima A, Wawrousek EF, Lobanoff MC, Charukamnoetkanok P, SmithGill SJ, Vistica BP, Lee RS, Egwuagu CE, Whitcup SM, Gery I: Immunotolerance against a foreign antigen transgenically expressed in the lens. Invest Ophthalmol Vis Sci 1998;39:2049-2057.

16 Ho WY, Cooke MP, Goodnow CC, Davis MM: Resting and anergic B cells are defective in CD28-dependent costimulation of naive CD4+ T cells. J Exp Med 1994;179: 1539-1549. 
-17 Kim SJ, Zhang M, Vistica BP, Chan CC, Shen DF, Wawrousek EF, Gery I: Induction of ocular inflammation by T-helper lymphocytes type 2. Invest Ophthalmol Vis Sci 2002;43: 758-765.

18 Fujimoto C, Yu CR, Shi G, Vistica BP, Wawrousek EF, Klinman DM, Chan CC, Egwuagu CE, Gery I: Pertussis toxin is superior to TLR ligands in enhancing pathogenic autoimmunity, targeted at a neo-self antigen, by triggering robust expansion of Th1 cells and their cytokine production. J Immunol 2006;177:6896-6903.
9 Chen J, Fujimoto C, Vistica BP, He J, Wawrousek EF, Kelsall B, Gery I: Active participation of antigen-nonspecific lymphoid cells in immune-mediated inflammation. J Immunol 2006;177:3362-3368.

0 Sakaguchi S, Ono M, Setoguchi R, Yagi H Hori S, Fehervari Z, Shimizu J, Takahashi T, Nomura T: Foxp3+ CD25+CD4+ natura regulatory $\mathrm{T}$ cells in dominant self-tolerance and autoimmune disease. Immunol Rev 2006;212:8-27.

21 Shevach EM, McHugh RS, Piccirillo CA, Thornton AM: Control of T-cell activation by CD4+ CD25+ suppressor T cells. Immunol Rev 2001;182:58-67.
2 Stockinger B: T lymphocyte tolerance: from thymic deletion to peripheral control mechanisms. Adv Immunol. 1999;71:229-265.

23 Choi S, Schwartz RH: Molecular mechanisms for adaptive tolerance and other T cell anergy models. Semin Immunol 2007;19: 140-152.

24 Weaver CT, Hatton RD, Mangan PR, Harrington LE: IL-17 family cytokines and the expanding diversity of effector $\mathrm{T}$ cell lineages. Annu Rev Immunol 2007;25:821-852. 